\title{
Erratum to: Real-time data driven deformation with affine bones
}

\author{
Byung-Uck Kim • Wei-Wen Feng • Yizhou Yu
}

Published online: 20 July 2010

(C) Springer-Verlag 2010

Erratum to: Vis Comput (2010) 26: 487-495
DOI 10.1007/s00371-010-0474-6

The author name below the title should be 'Wei-Wen Feng' instead of 'Wei-Wei Feng'.

The same correction also applies to the author name in the biography on page 495 .
The 13th line in Abstract should be 'a two-layered regression' instead of 'a two-layer regression'.

The 7th and 8th lines of the second paragraph in Sect. 3.3, on page 490, should be 'rotation $\mathbf{Q}$ ' and ' $\mathbf{F}=\mathbf{Q W}$ ' instead of 'rotation $\mathbf{R}$ ' and ' $\mathbf{F}=\mathbf{R W}$ ' respectively.

The same correction also applies to the 8th and 9th lines of the second paragraph in Sect. 5 on page 491.

The online version of the original article can be found under doi:10.1007/s00371-010-0474-6.

B.-U. Kim (凶) · W.-W. Feng · Y. Yu

Department of Computer Science, University of Illinois at Urbana-Champaign, 201 N. Goodwin Ave., Urbana, IL 61801, USA

e-mail: kbu@illinois.edu

W.-W. Feng

e-mail: wfeng2@illinois.edu

Y. Yu

e-mail: yyz@illinois.edu 